\title{
FIRE EVACUATION SIMULATION CONSIDERING THE MOVEMENT OF PEDESTRIAN ACCORDING TO FIRE SPREAD
}

\author{
Jaeyoung Lee, Minhyuck Lee, Chulmin Jun *
}

Dept. of Geoinformatics, University of Seoul, Korea, - \{ljyda214, Imhll123,*cmjun $\} @$ uos.ac.kr

KEY WORDS: Fire evacuation simulation, Floor field model, Fire dynamics simulator, Indoor evacuation, Fire spread field, Fire recognition field

\begin{abstract}
:
A fire simulator and an evacuation simulator are generally used independently to diagnose the safety of a building in the case of the fire and evacuation. However, it is hard to provide highly accurate safety diagnosis with this method because it does not reflect the movement of pedestrians in the situation of a fire. Therefore, this study proposed a fire evacuation simulation technique that can describe the movement of pedestrians with considering the fire spread. The proposed simulation technique applies the fire spread data of the fire dynamics simulator (FDS) to the floor field model (FFM) and it models that pedestrians recognizes the fire and take a detour to a safe route. This study proposed a method to link the data between FDS and FFM and an improved FFM considering fire spread. Additionally, the proposed method was applied to a real building on a university campus. This study simulated evacuations under various scenarios. Simulation results showed that the number of evacuees escaping through each exit varied by the presence of fire. Moreover, it was found that the evacuation time was increased or decreased by the fire and bottleneck phenomenon was also worsened under fire situation.
\end{abstract}

\section{INTRODUCTION}

A fire simulator and an evacuation simulator are generally used independently to diagnose the safety of a large building in the situation of fire and evaluation. An evacuation simulator is used to estimate the required safe egress time (RSET), which is the time required for people in the building to move to a safe location on foot. a fire simulator is used to calculate the available safe egress time (ASET) which is time before the fire affects pedestrians. The safety of a building is diagnosed by comparing these two indices (Kim and Jeon, 2015). Pathfinder, Simulex, and buildingEXODUS are representative evacuation simulators and FDS, CFAST, and Smartfire are widely used by fire simulators.

However, it is difficult to accurately and practically diagnose the actual safety of a building using a fire simulator and an evacuation simulator independently because this method does not reflect the movement of pedestrians under a fire spreading situation. When a pedestrian finds a risk factor such as smoke or fire on the moving route, he or she will detour through a safer route. However, conducting a fire simulator and an evacuation simulator independently means that the description of fire spread and the description of pedestrian's movement are not linked. As a result, in an independently conducted evacuation simulation, pedestrians did not choose a detour even if there was a fire and pedestrians required to take a detour. Various studies have been conducted to overcome this limitation. Studies have developed into a form that visualizes fire spread and the visualized fire spread influences the movements of pedestrians by linking two simulators, rather than simply expressing the fire status in an evacuation simulator. BuildingEXODUS and FDS+Evac are representative simulators showing this model linkage. However, it is hard to obtain BuildingEXODUS because it is not an open source program.
Moreover, FDS+Evac has a shortfall that the calculation speed lowers drastically when there are many pedestrians and the structure of a building is complex. It means that it takes a long time to run an evacuation simulation for a large building.

Therefore, this study proposed a fire evacuation simulation that would be able to describe the movement of pedestrians with considering the fire spread in the condition of many pedestrians and complex building structure by using FFM, known to have fast calculation speed. The proposed fire evacuation simulation was to describe the evacuation situation by considering fire spread by applying the fire spread data of FDS, a fire simulator, with FFM, a pedestrian model. The study consisted of the data linkage between FDS and FFM and the improved FFM development with considering fire spread. The experiment of the proposed methodology was conducted by using EgresSIM (Nam et al., 2016b), an evacuation simulator.

Chapter 2 will describe FFM and FDS. Moreover, Chapter 2 will review the existing literature related to fire evacuation simulation studies and the uniqueness of this study compared to the previous studies. Chapter 3 will describe the data linkage and visualization methods of FDS and FFM. Chapter 3 will also explain the development of the improved FFM, reflecting the effects of fire spreading, more specifically. Chapter 4 will describe the analysis results of simulation for campus building. Chapter 5 will discuss the conclusions of this study.

\section{LITERATURE REVIEW}

\subsection{Floor field model}

FFM is a pedestrian model, which was first introduced in 2001, and it models the micro-scale movement of pedestrians (Burstedde et al., 2001). FFM is based on a two-dimensional

\footnotetext{
* Corresponding author
} 
CA model and factors affecting the movement of pedestrians are presented in the form of a lattice floor field. A pedestrian is placed in a lattice shape cell and the pedestrian determines the location of the next cell through the interaction with the surrounding eight cells (Figure 1). The pedestrian moves to the target area by repeating this process. The distance to an exit and the interaction with neighboring pedestrians affect the movement of a pedestrian. FFM describes the overall evacuation situation by integrating the conditions of the floor fields.

Static floor field (SFF) indicates how easy a pedestrian can move to an exit from each cell and SFF is assigned to each cell. The distance to the exit is generally used for variable of SFF. Dynamic floor field (DFF) is also assigned to each cell and it indicates the interaction between neighboring pedestrians. It means the attraction and repulsion effects between one pedestrian and another pedestrian. Figure 2 shows the structure of FFM consisting of spatial data and two floor fields. A pedestrian determines the next cell to move by calculating the SFF and DFF values of surrounding cells at each time step. Additionally, FFM can simulate various situations such as a darkness condition by adjusting the sensitivity parameter of the field (Kirchner et al., 2002).

\subsection{Fire dynamic simulator}

FDS is a fire simulator created by the National Institute of Standards and Technology (NIST) for analyzing the heat and smoke due to fire (Kim et al., 2013). Input variables of FDS are the target space of fire simulation, fire size, the geometrical shapes of indoor objects (e.g., walls, floors, furniture, and others), the attributes of indoor objects (e.g., material, density, and heat conduction), ventilation condition (e.g., ventilator and air circulation), the devices for extinguishing fire (e.g., sprinkler), and analysis parameters. It expresses the space where fire occurs and objects in the space based on input variables.

Fire is implemented by the attributes of fire, which were entered for the expressed space and objects (McGrattan et al., 2013). The heat and smoke spread of the implemented fire are calculated based on computational fluid dynamics (CFD) and large eddy simulation (LES) is widely used as a turbulence model. The flow of combustion products and heat transfer is calculated numerically by using the Navier-Stokes equation. For the qualitative analysis of this, FDS uses SmokeView (SMV), a visualization tool, to visualize the information of various parameters and compare them in a space according to time change. Moreover, FDS uses fds2ascii to print out the quantitative spatial information visualized by SMV as a text format for quantitative analysis. (Rie, 2008). The figure 3 shows the fire simulation in a virtual building simulated by FDS and visualized by SMV.
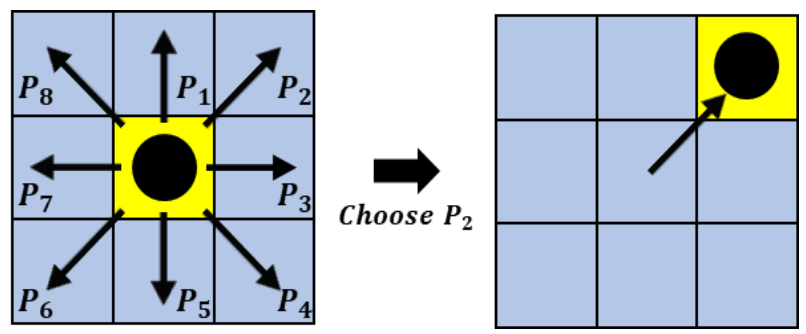

Figure 1. Movement of an agent in FFM

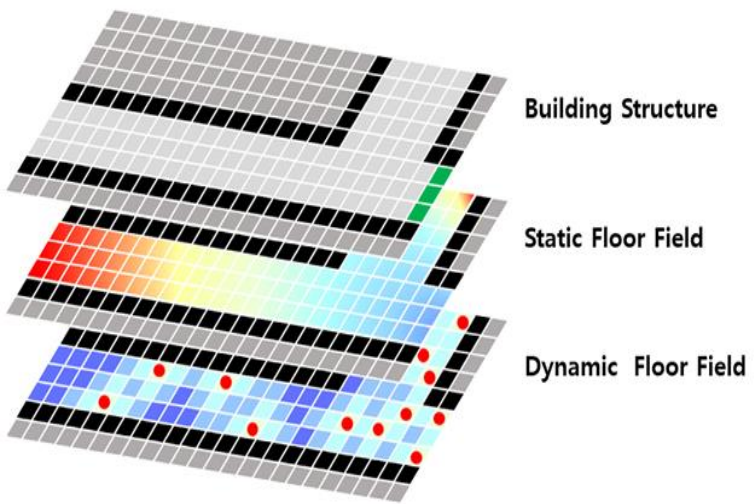

Figure 2. The structure of FFM

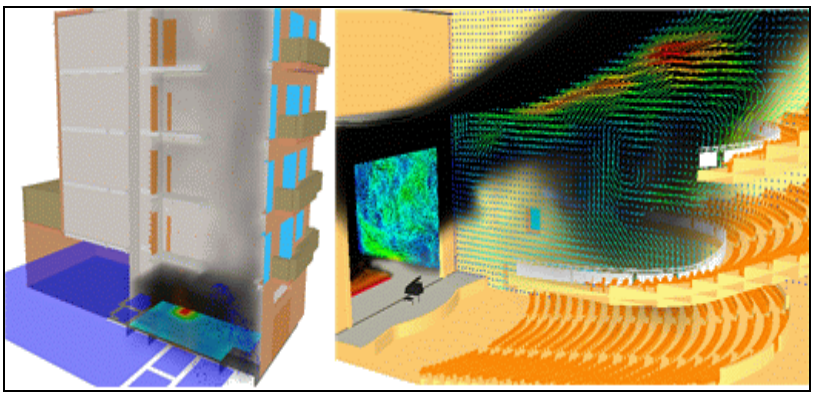

Figure 3. Simulation of FDS

\subsection{Fire evacuation simulation}

The method of using an evacuation simulator and a fire simulator can be divided into three types: non-coupling, semicoupling, and coupling depending on the level of coupling between the two simulators (Koo, 2017). The non-coupling method, as stated in the introduction, conducts a fire simulator and an evacuation simulator independently to estimate ASET and RSET for a certain point and compare the results It is a commonly used in South Korea (Choi, 2011). The building stability assessment using a non-coupling method compared RSET and ASET directly. For example, when RSET is larger than ASET, it is interpreted that fire can affect the people in the building before they can evacuate, which means that the structure of the building is dangerous. On the other hand, when RSET is smaller than ASET, it concludes that people can complete the evacuation without being affected by the fire. Pathfinder and FDS are representative Non-coupling type simulators. However, the non-coupling method cannot evaluate the evacuation of people in the fire situation appropriately because it excludes the response of people to fire. The semicoupling method checks the fire spread and the movement of pedestrians at the same time. However, the semi-coupling method simply superimposes the fire spread with the movement of pedestrians so the fire does not affect the behaviors of pedestrians. Consequently, this method also has limitations to describe the evacuation realistically (Koo et al., 2017). In the coupling method, the fire influences the behavior of pedestrians. Therefore, it can simulate the behaviors of pedestrians realistically. BuildingEXODUS and FDS+Evac are representative coupling type simulators.

FDS+Evac uses the social force model (SFM), which describes the factors affecting the movement of pedestrians as force, expressed as vectors, and shows the movement of pedestrians by calculating the sum of vectors (Korhonen et al., 2008). 
FDS+Evac uses the exit selection algorithm by using the smoke concentration among the fire analysis results of FDS. The exit selection algorithm excludes an exit, which is not visible due to smoke, from the simulation (Korhonen and Hostikka, 2010). SFM has a high calculation complexity so it requires high computation time when there are many pedestrians and the structure of a building is complex (Nam, 2012). Since FDS uses $\mathrm{CFD}$, the computation time increases exponentially when the size of a building increases or the structure of a building becomes more complex. FDS+Evac uses the two models and, consequently, even a minor scenario change (e.g., relocation of people) can drastically increase the computation time.

\subsection{Differentiation from related studies}

This study proposed a coupling method fire evacuation simulation that couples FDS and FFM. FFM calculates fast because an agent, which refers to a pedestrian, determines the movement by considering only eight adjacent cells. However, it can also become a disadvantage because the agent cannot notice the dangerous situation until the fire (e.g., heat and smoke) arrives at adjacent cells (Lee et al., 2017). Therefore, this study improved FFM so that agent could recognize fire even not adjacent to the fire and could detour to a safer exit.

\section{METHODOLOGY}

The fire evacuation simulation methodology using FFM and FDS is composed of two parts. The first is to link data to reflect the fire spread data of FDS to a pedestrian model, FFM. The data not only contain the fire spread data but also include the spatial data of FDS and FFM. The second is to propose the improved FFM so the model can consider the fire spread based on the linkage with FDS. The improved FFM has two algorithms for agents, one is to recognize the fire and the other is to detour to a safe exit when the fire is recognized.

\subsection{Data linkage}

Data linkage is composed of two processes. The first is the process of converting the spatial data of FFM to the spatial data of FDS to conduct a fire simulation. The second is to reflect the fire spread data computed from FDS to FFM. The process is summarized in Figure 4.

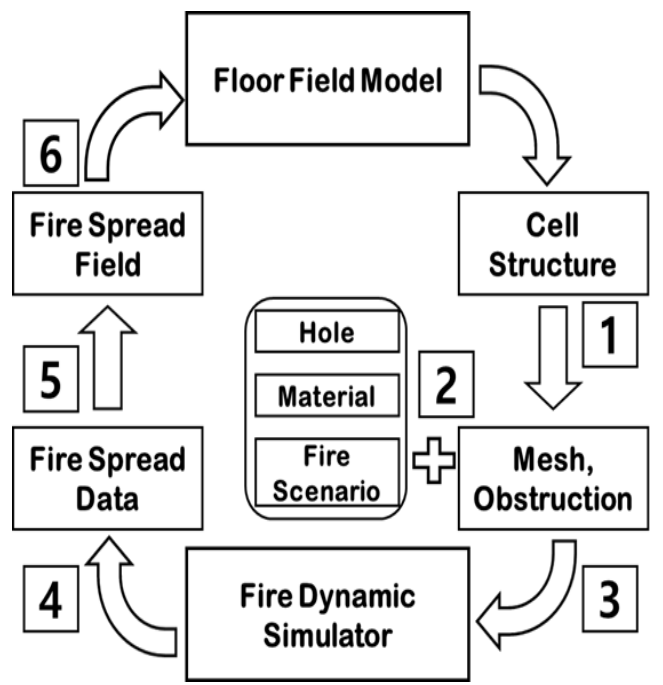

Figure 4. Data linkage in FFM and FDS

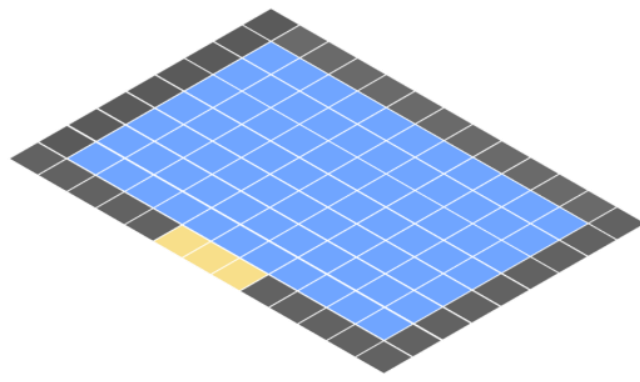

(a) Cell structure of FFM

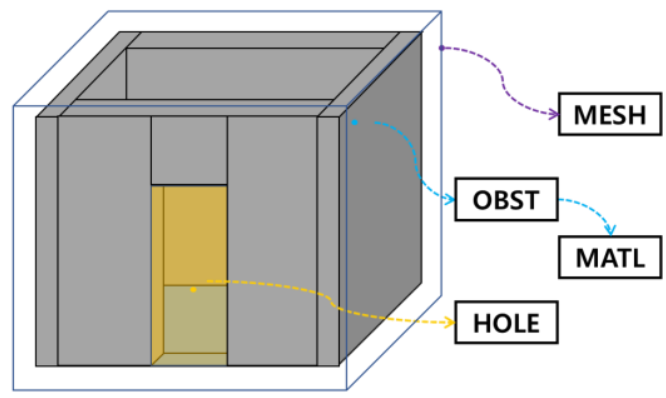

(b) Voxel structure of FDS

Figure 5. Spatial data structure

\subsubsection{Spatial data conversion}

The spatial data of the FFM consists of a group of individual cells. Each cell is a square shape $(0.3$ or $0.4 \mathrm{~m}$ wide $)$ considering the stride of the agent (Figure 5-a). There are two types of cells (i.e., non-walkable cells (e.g., wall and obstacle) and walkable cells). FDS uses 3D spatial data (Figure 5-(b). Spatial data of FDS is composed of MESH, OBST, HOLE, and MATL, which represent fire analysis space where fire is simulated, rectangular shape objects to express wall, furniture, and obstacles, empty space between objects or space passing through a well such as a window or a door, and the attributes of a material compositing an object, respectively. The fire analysis space is subdivided by using voxels and each voxel means the resolution to be applied by a user to interpret the simulation results.

The FFM of this study utilizes $30 \mathrm{~cm}$ size cells (Nam et al., 2016a). Therefore, the height, width, and depth of FDS's voxel were defined as $30 \mathrm{~cm}$ each. The fire analysis space was created to be equal to the minimum bounding box (MBB) of the target building. Rectangular objects, which express the physical structure of the indoor space, were created by expanding each cell of FFM to the height of the target building's level. Lastly, although it is not considered in FFM, this study added a window and a door (Hole) and the attributes of materials (MATL) composing objects to complete data conversion.

\subsubsection{Fire spread field}

It is possible to extract fire spread data by inputting the location of fire start and the size of the fire to FDS along with the converted spatial data. The fire spread data contains the heat, smoke concentration, and visibility for each voxel at each time step. This study added a fire spread field to FFM by applying the data to FFM in order to visualize the fire spread and to help agents recognize it. 

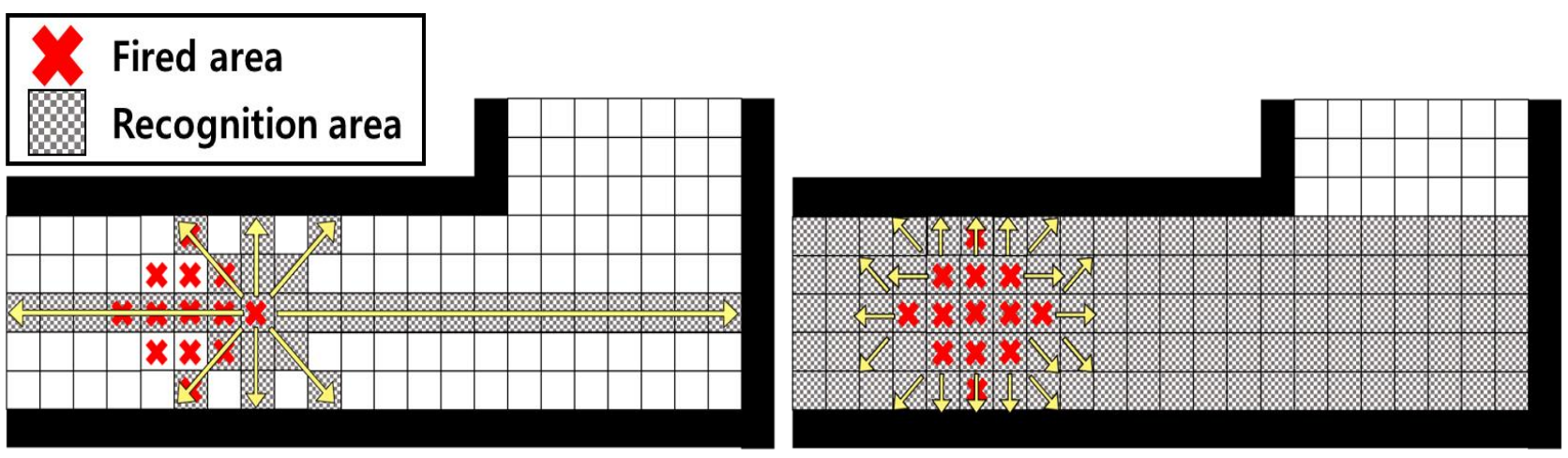

Figure 6. A process of fire recognition field computation

The fire spread field refers to the fire spread data at a certain height. The spatial range is a group of voxels at a certain height. In other words, the spatial range is a set of two-dimensional planar cells that store heat, smoke concentration, and visibility values. When conducting FDS, a user inputs a time interval for analyzing results. The fire spread data show the results by the voxel unit according to the time interval. Therefore, if the fire spread data is collected $\mathrm{N}$ times for the entire simulation, the fire spread field will be renewed $\mathrm{N}$ times as well.

\subsection{FFM considering fire spread}

In FFM, the agent moves with only considering eight adjacent cells. It decreases the computational complexity, which is an advantage, but it is an inherent limitation that the agent cannot recognize fire until it is in a directly adjacent cell. If the risk due to the fire is assigned to the whole space like SFF, it will be possible to realize the walking with considering the fire (Zheng et al., 2017; Wang and Wang, 2016). However, it means that the agent can move by recognizing the condition of the whole space and it cannot be a realistic pedestrian model. This study assumed that "A pedestrian takes a detour when the person recognizes a risk factor in the moving direction" and described the evacuation situation considering fire spread by using the following two algorithms.

\subsubsection{Fire recognition algorithm}

The fire recognition algorithm is an algorithm that calculates the space where agents can recognize the fire. The space is called a fire recognition field and it is calculated separately. A pedestrian entering the fire recognition field selects a detour. The fire recognition field is calculated in the following order and Figure 6 shows a process of Fire Recognition Field computation.

Step 1. Select all the cells where the heat and smoke exist in the fire spread field.

Step 2. For one of the selected cells, identify all walkable cells that exist in the eight-way direction (i.e., E, SE, S, SW, W, NW, $\mathrm{N}$, and NE) for any one of the selected cells and add them to the fire recognition field.

Step 3. Repeat the Step 2 for all the cells selected in Step 1.
Figure 7 shows the structure of the improved FFM that includes the structure of the existing FFM and the fire spread field and the fire recognition field, which were generated through the above processes.

\subsubsection{Detour algorithm}

An agent entering the fire recognition field take a detour to an exit, which has the minimum risk factors and the shortest travel distance. The detour algorithm describes this process and it uses the graph data structure. The improved FFM proposed in this study uses that graph data in order to apply the detour algorithm to agents in addition to the basic cell data.

The graph data form a hierarchical relationship with the cell data (Figure 8). The target space was divided into subdivisions and each subdivision becomes one node. Cells located in the subdivided space become a sub-object (parent-child dimension) of the node, which means the corresponding space. The edge, which means the connectivity between the nodes, stores the distance between nodes. The value is the distance between the center cell of each node.

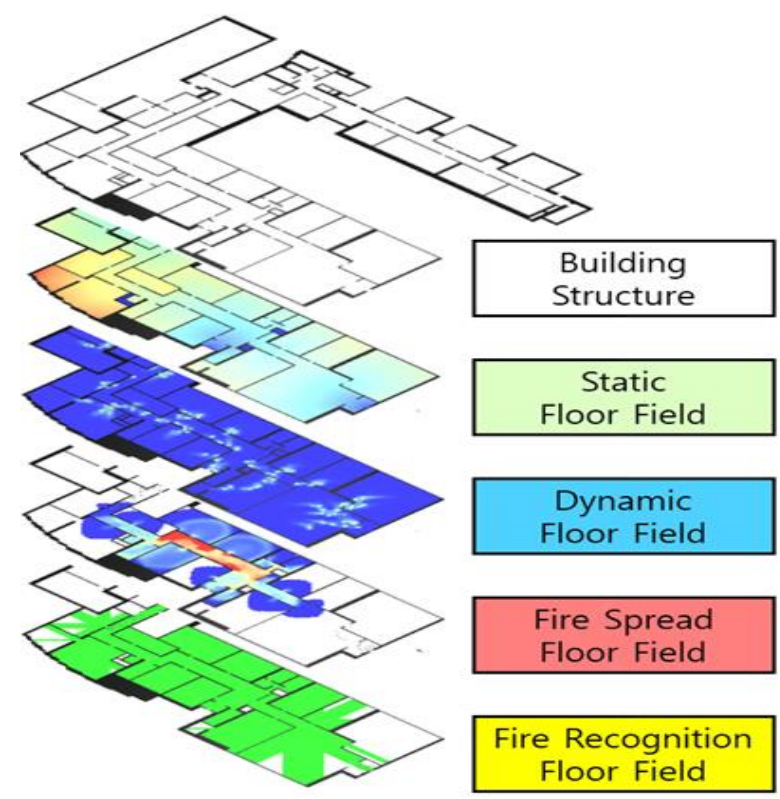

Figure 7. Structure of the improved FFM

Step 4. When the fire spread field is renewed, repeat from the Step 1 to renew the fire recognition field. 


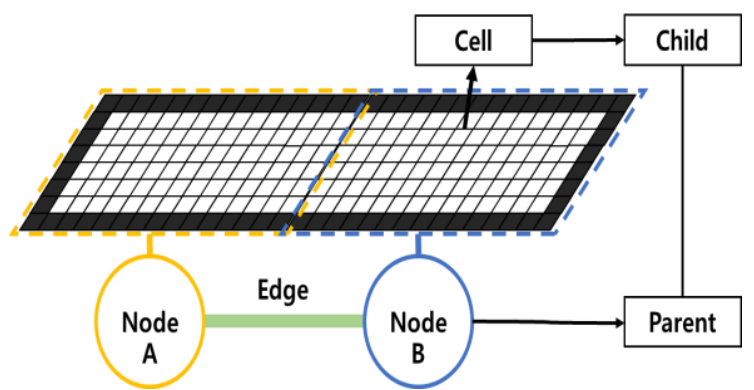

Figure 8. Hierarchical data structure of the improved FFM

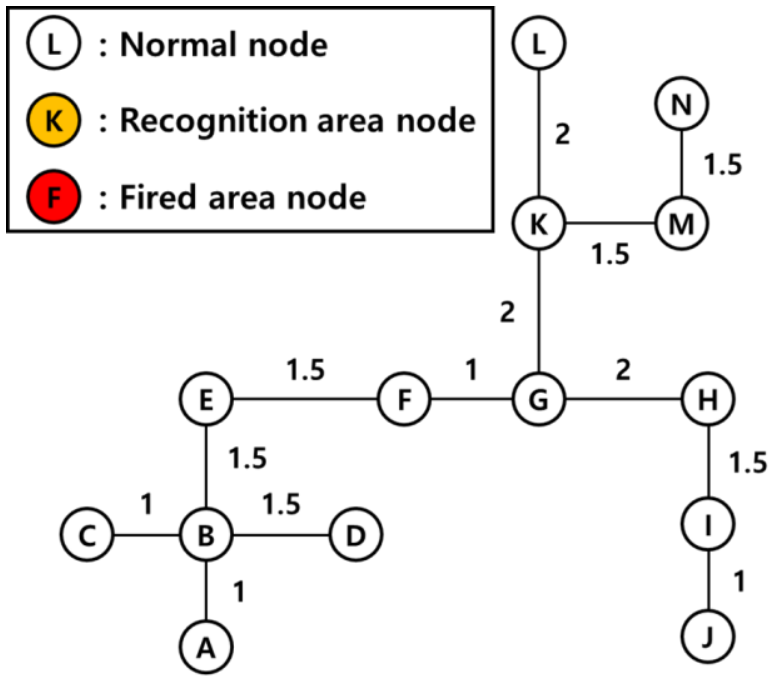

(a) Ordinal graph network

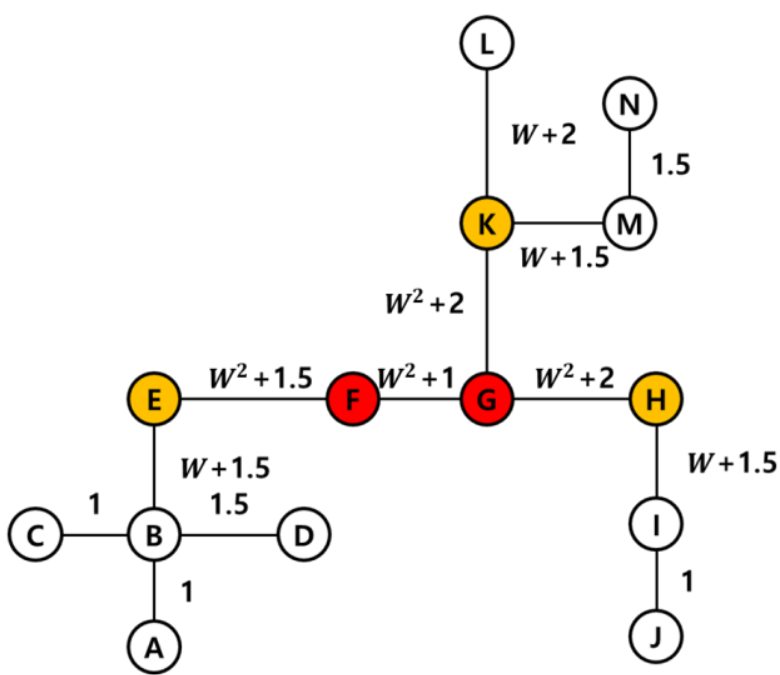

(b) graph network in fire situation

Figure 9. Graph network without and with fire

When a node has heat or smoke (fire spread field) or is located in a fire recognition field, this study added weights to the connectivity to adjacent nodes. Edges connecting safe nodes store only distance, while edges located in a dangerous space or showing connectivity with a dangerous space stores a weight in addition to a distance. When the weight of an edge belonging only to a fire recognition field is $\mathrm{w}$, the weight of the edge belonging to a fire spread field is w2. It was to distinguish between the case of just recognizing the fire and the case of affected by the fire. A very large value, close to infinity, is assigned to w2. When the sum of distance and weight is equal to the evacuation cost, the evacuation cost is renewed along with the fire spread field. The figure 9 shows Graph network without and with fire.

Based on the described data structure, when an agent entering in a fire recognition field is observed, an exit having the minimum evacuation cost is explored with using the node where the agent is located as a starting point by using Dijkstra algorithm. When an exit is found, the SFF of the agent is renewed to only have the found exit. This algorithm forces the agent to use the found exit as a destination. It describes a pedestrian who select the second-best option when the initially identified exit is no longer available.

\section{EXPERIMENT}

\subsection{Fire evacuation simulation using EgresSIM}

This study realized a fire evacuation simulation based on FDS and FFM by using 'EgresSIM', an evacuation simulator (Nam et al., 2016b). EgresSIM is microscopic evacuation simulator. It is a three-dimensional (3D) pedestrian evacuation simulator based on FFM. This simulator can simulate large size buildings that consist of a number of floors, stairs, rooms, and exits placing several hundreds or thousands of pedestrians in test space. It can check their movements through the 3D viewer in real time. Moreover, it shows detailed results about evacuation situations such as which paths are employed by individual pedestrians, how long does it take to evacuate, and how many evacuees escape at each of the exit doors.

The development process was composed of the addition of an interface for the data linkage between FDS and EgresSIM, the development of a fire spread field and a fire recognition field visualization, and the implantation of the improved FFM. This experiment was a fire evacuation simulation using EgresSIM. This study analyzed the difference between the evacuation results with and without fire. 


\subsection{Scenarios}

Figure 10 shows the target space and the location of the fire to be simulated. The target space was a first floor of actual building structure with five exits and it was assumed that 300 people were distributed in the space evenly. Two fire scenarios were tested: ignition at location 1 and ignition at location 2 . The Heat Release Rate Per Area (HRRPUA) of two cases were identical $(100 \mathrm{Kw} / \mathrm{m} 2)$.

\subsection{Simulation results and analysis}

Simulations were conducted under three situations based on scenarios. Situation 1 was a case without a fire. Situation 2 was when a fire initiated at location 1 . Situation 3 was when a fire initiated at location 2. Figures 11 and 12 show simulations of Situation 2 and Situation 3, respectively. It shows the fire spread over time and people evacuated by avoiding the fire.

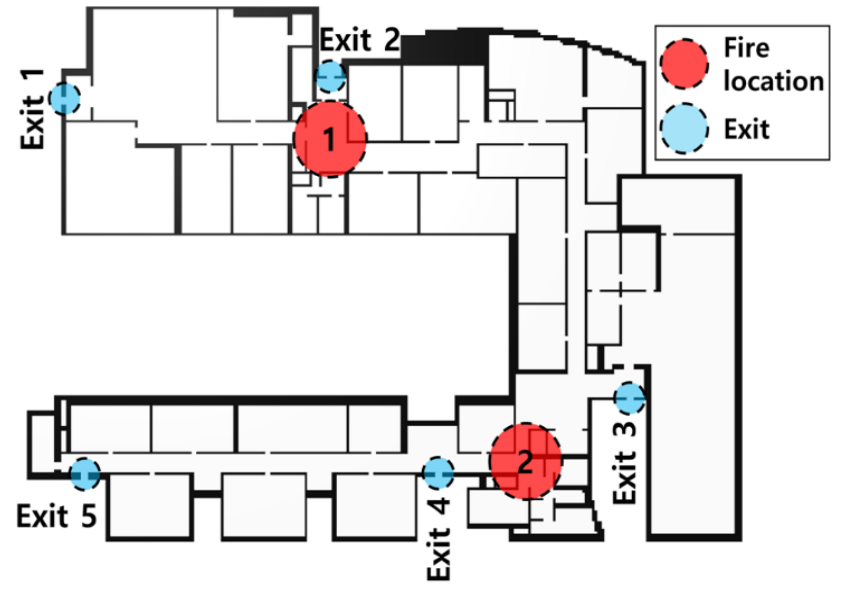

Figure 10. The test space with fire locations

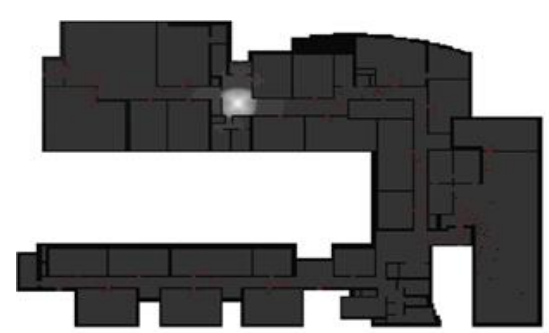

Time $=15$

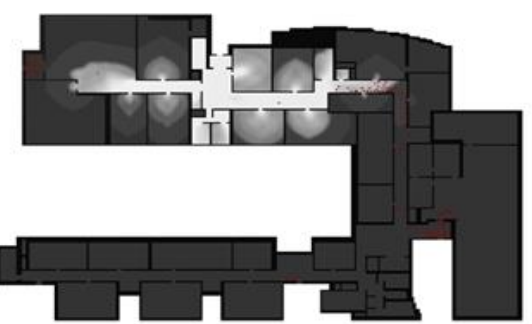

Time $=60$

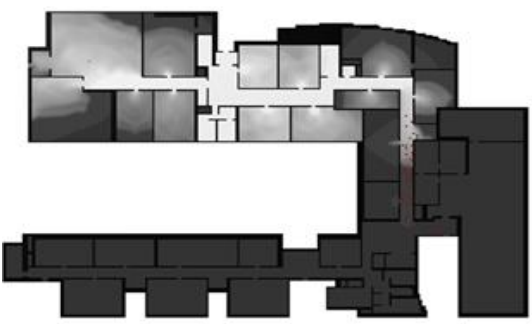

Time $=105$

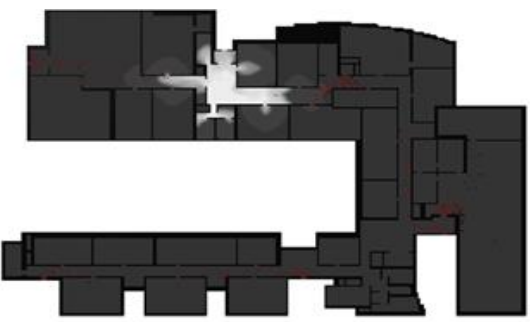

Time $=30$

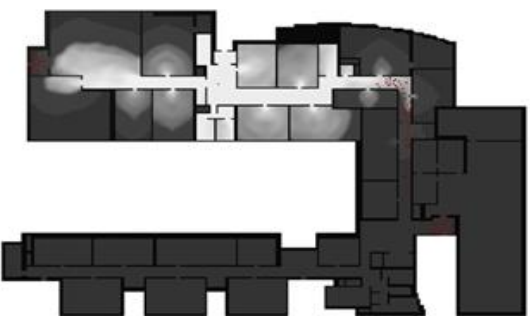

Time $=75$

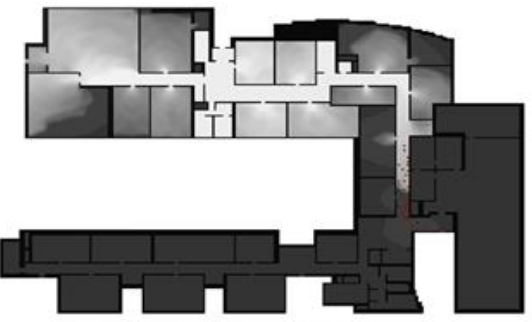

Time $=120$

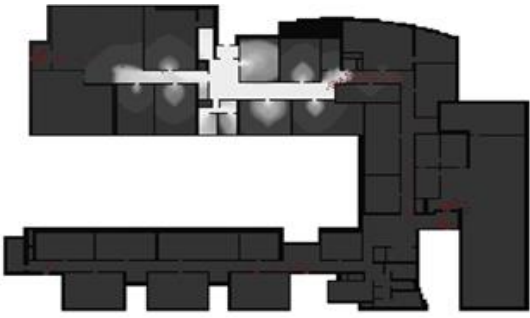

Time $=45$

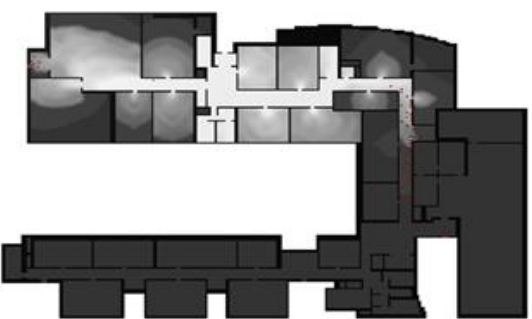

Time $=90$

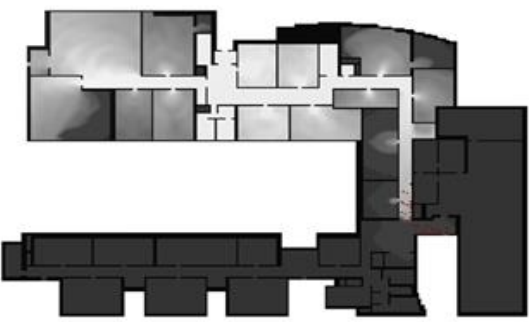

Time $=135$

Figure 11. The simulation on situation 2 


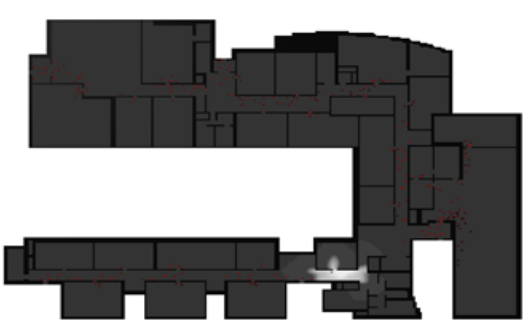

Time $=15$

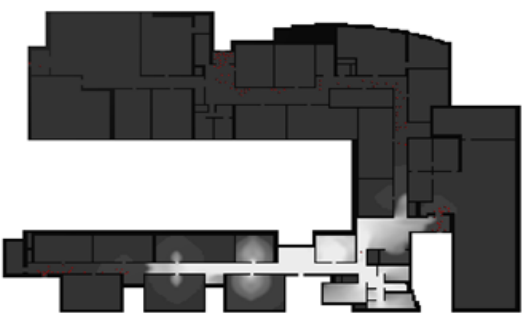

Time $=60$

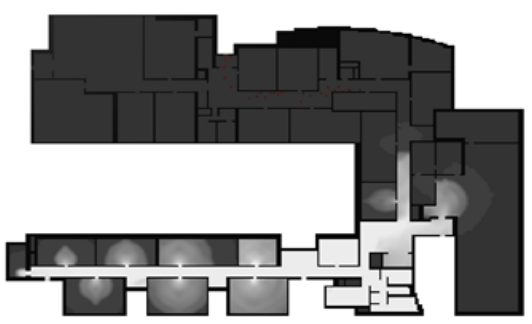

Time $=105$

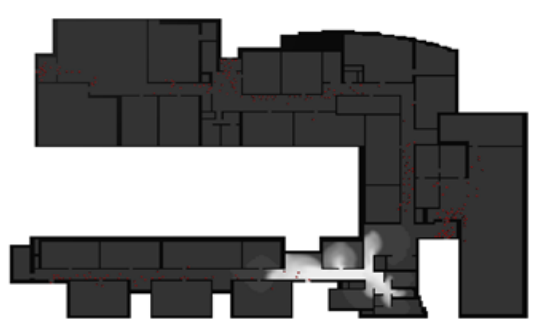

Time $=30$

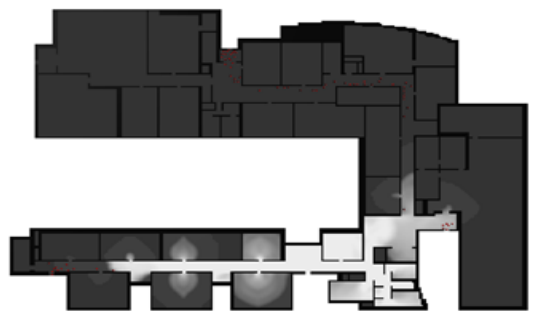

Time $=75$

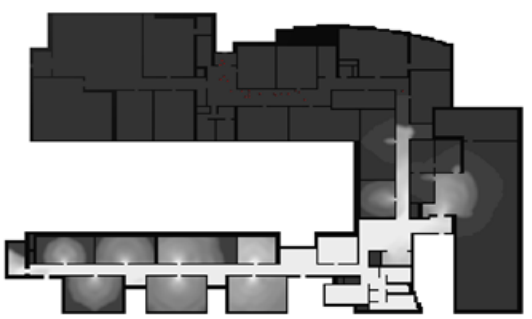

Time $=120$

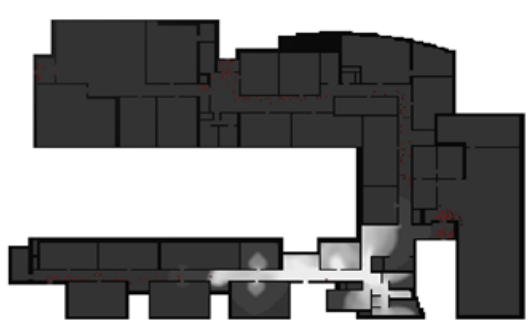

Time $=45$

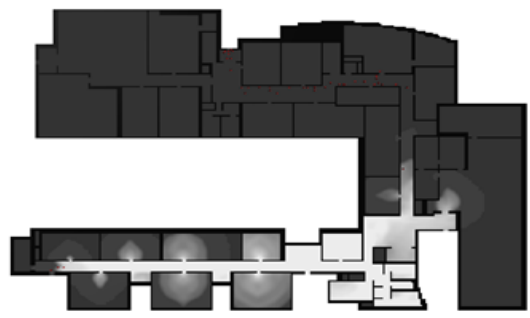

Time $=90$

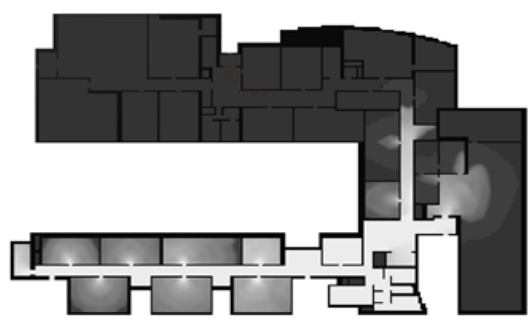

Time $=135$

Figure 12. The simulation on situation 3

Figures 13, 14, and 15 indicate the cumulative evacuees at each time interval for each exit and the trajectories of pedestrians at each situation. When there was no fire (situation 1), people mostly escaped through Exit 2 and 3 under the given distribution assumption. As a result, the evacuation at Exits 1, 4, and 5 ended within 70 seconds, while the evacuation at Exits 2 and 3 ended at approximately 125 seconds.

When a fire occurred near Exit 2 (situation 2), people who headed to Exit 2 detoured to Exit 1 and 3. As a result, compared to situation 1, the number of evacuees increased by 26 and 50 at Exit 1 and 3, respectively, while the number of evacuees at Exit
2 decreased by 75 . The evacuation time at Exit 2 is reduced by 94 seconds, but the evaluation time at Exit 1 and 3 increased by 32.5 and 43 seconds, respectively. The results showed that people collided with each other while they tried to take a detour from Exit 2 to Exit 3 and, consequently, the evacuation time increased drastically. Moreover, a bottleneck phenomenon was found at Exit 3 even when there was no fire. The bottleneck phenomenon became more serious when a fire occurred nearby Exit 2. Therefore, it is believed that a strategy to detour evacuees to Exit 4 is needed.
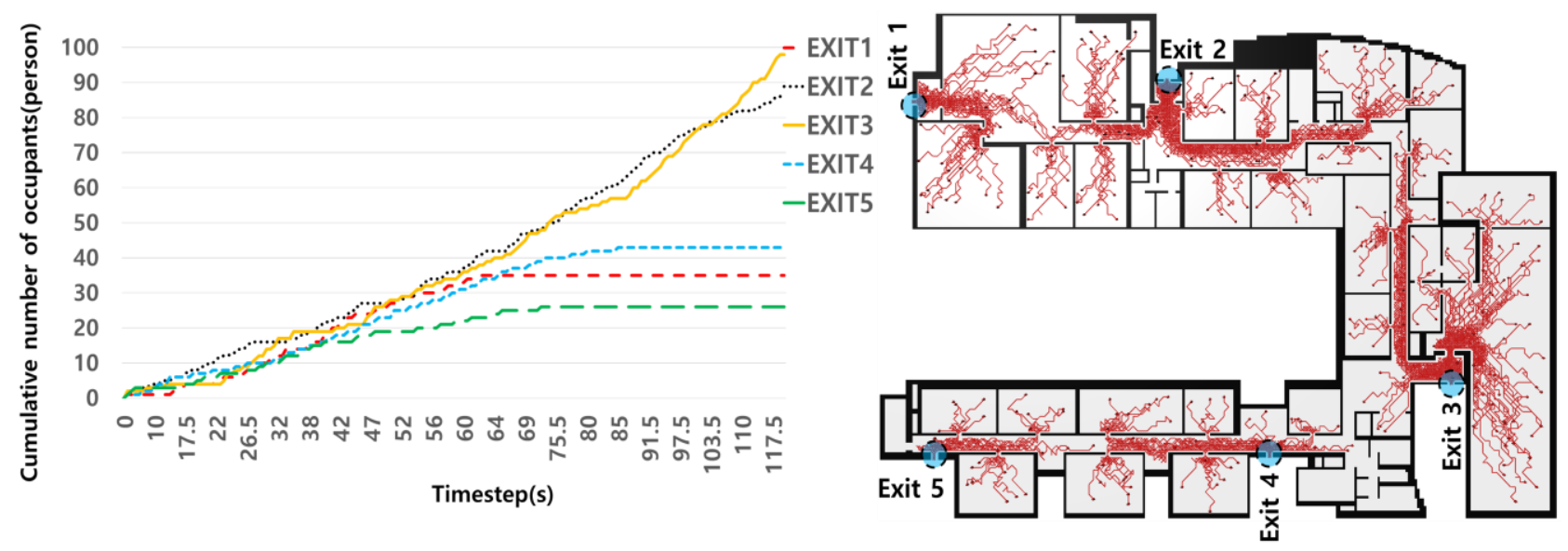

Figure 13. Situation 1: Evacuation without a fire 

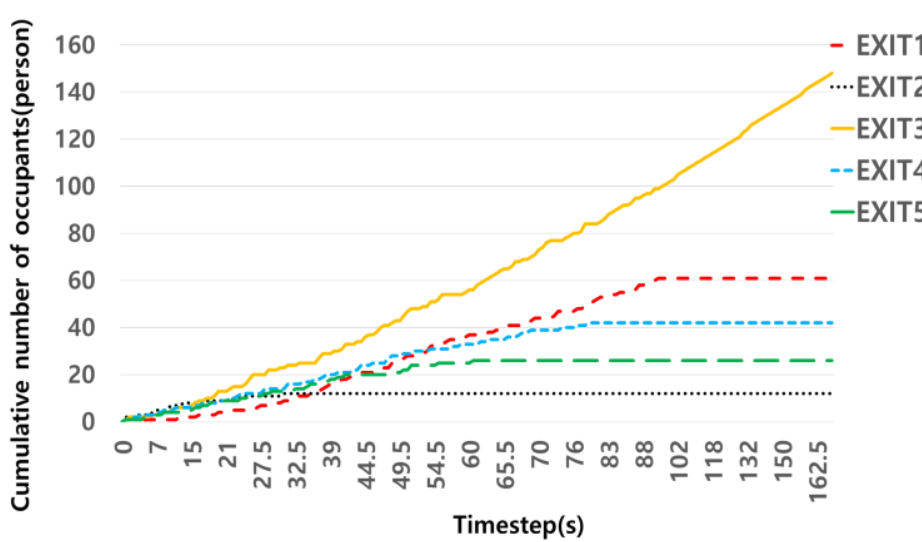

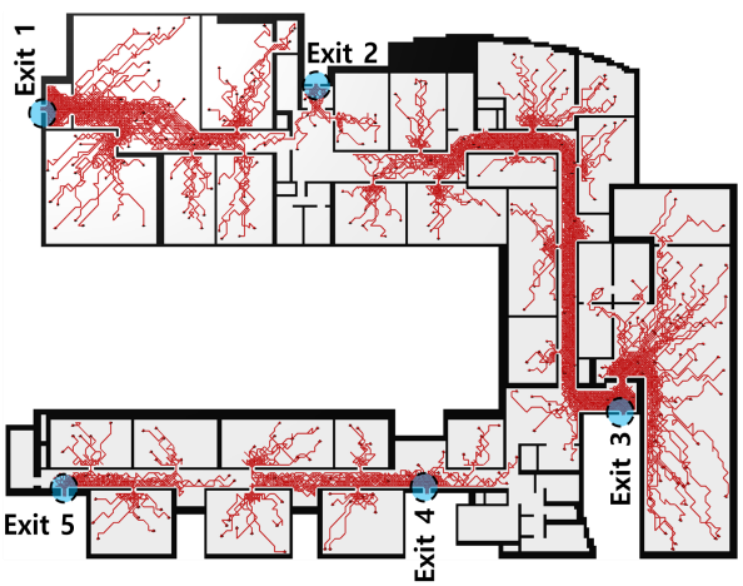

Figure 14. Situation 2: Evacuation when a fire initiated at location 1

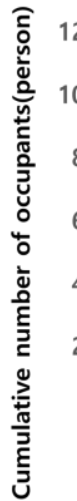

120
100
80
60
40
20
0
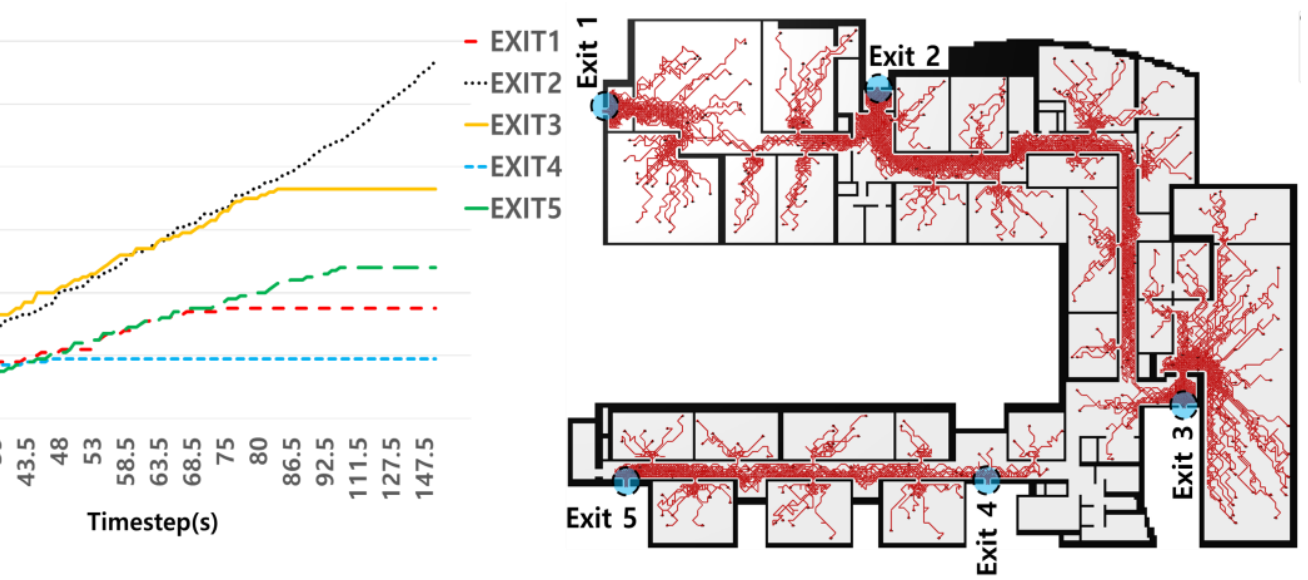

Figure 15. Situation 3: Evacuation when a fire initiated at location 2

When a fire occurs near Exit 3 (situation 3), more evacuees went to Exit 2 and 5. Compared to situation 1, 27 more evacuees escaped through Exit 2 and 25 fewer evacuees evacuated through Exit 3. Exit 4, closest to the fire, had 24 fewer evacuees and 22 more evacuees went to Exit 5 . Consequently, the evacuation time decreased by approximately 58 seconds at Exit 3 and increased by approximately 26 seconds at Exit 2 . It decreased by approximately 26 seconds at Exit 4 and it increased by approximately 28 seconds at Exit 5 . The overall evacuation completion time was approximately 152 seconds. Exit 2 showed a bottleneck phenomenon even when there was no fire. In situation 3 , the bottleneck phenomenon was worsened. Therefore, it is necessary to have a strategy to move evacuees heading to Exit 2 toward Exit 1. Since evacuation ended at approximately 75 seconds at Exit 1, if the evacuees concentrated at Exit 2 could move to Exit 1, the overall evacuation completion time will be shortened by reducing the evacuation time at Exit 2.

\section{CONCLUSIONS}

This study proposed a fire evacuation simulation with coupling FDS and FFM. The fire spread data of FDS were converted into the cell structure and reflected to FFM. Based on this modification, this study developed the improved FFM considering fire spread. The improved FFM generates a fire spread field based on the fire spread data of FDS. Moreover, it generates a fire recognition field using a fire spread field for an agent to recognize a fire. Thereafter, a detour algorithm, which changes the route of an agent when the agent enters a fire recognition field to avoid fire, was applied to the agent.

This study simulated fire evacuation simulations using the improved FFM with and without the occurrence of fire. When comparing the results of with and without fire situation, the number of evacuees at each exit varied a lot due to the detour of agents. As a result, the evacuation time of each exit clearly increased or decreased, and the bottleneck phenomenon was clearly worsened as well.

The fire evacuation simulation proposed in this study described the evacuation situation more realistically than the existing method that conducts a fire simulation and an evacuation independently and separately. Moreover, the proposed FFM can quickly calculate the evacuation simulations under various population distribution conditions. This study had limitations of not reflecting the psychological or behavioral patterns of pedestrians in the fire situation and applying only one assumption that evacuees unconditionally detoured when they recognized a fire. Additionally, this study assumed that the fire recognition field was created for eight directions from the cell in which heat and smoke are present. Therefore, it would take different amount of time for pedestrians to recognize the fire, even though they are in one space. It could be another limitation of this study. It is expected that a more realistic evacuation simulation will be developed if the identified limitations are supplemented in future studies. 


\section{ACKNOWLEDGEMENTS}

This research was supported by a grant (18NSIP-B135746-02) from National Spatial Information Research Program (NSIP) Program funded by Ministry of Land, Infrastructure and Transport of Korean government.

\section{REFERENCE}

Burstedde, C., Klauck, K., Schadschneider, A., and Zittartz, J., 2001. Simulation of pedestrian dynamics using a twodimensional cellular automaton. Physica A: Statistical Mechanics and its Applications, 295(3-4), pp. 507-525.

Choi, J.H., 2011. Development of performance based egress design and modeling method through analyzing evacuation characteristic of a high-rise building. Ph.D.Thesis, Kyungpook National University, Daegu, Korea.

Kirchner, A. and Schadschneider, A., 2002, Simulation of evacuation processes using a bionics-inspired cellular automaton model for pedestrian dynamics, Physica A: Statistical Mechanics and its Applications, 312(1-2), pp.260276

Kim, J.Y., and Jeon, Y.H., 2015. The case analysis through fire simulation FDS and evacuation simulation pathfinder. AsiaPacific Journal of Business Venturing and Entrepreneurship, 10(6), pp. 253-260.

Kim, M.G., Joo, Y.J., and Park, S.H., 2013. Risk evaluation and analysis on Simulation Model of Fire Evacuation based on CFD - Focusing on Incheon Bus Terminal Station. Journal of Korea Spatial Information Society, 21(6), pp. 43-55.

Koo, H.M. 2017. A Study on Improvements Performance-Based Design Standards through a Comparsion of Life Safety Assessment Results according to Three Coupling Methods of Fire and Evacuation Simulation. MA, Pukyong National University. Pusan, Korea.

Koo, H.M., Oh, R.S., Lee, S.H., Ahn, S.H., Hwang, C.H., Choi, J.H., 2017. A Comparative Study on Life Safety Assessment Results depending on Whether Coupling of Fire \& Evacuation Simulation for Performance Based Design. Proceedings of 2017 KIFSE Annual Spring Conference. pp.47-48

Korhonen, T. and Hostikka, S., 2010. Fire Dymanics Simulator with Evacuation: FDS+EVAC Technical Reference and User's Guide. VTT Research Note, VTT, Finland.

Korhonen, T., Hostikka, S., Heliövaara, S., and Ehtamo, H., 2008. FDS+Evac: An Agent Based Fire Evacuation Model .4th Intl. Conference on Pedestrian and Evacuation Dynamics. Germany, Wuppertal, Feb. 27-29, 2008, pp.109-120.

Kwak, S.Y., Nam, H.Y., and Jun, C.M., 2016. Microscopic Evacuation Simulation in Large-scale Buildings using EgresSIM. Journal of the Korea Society for Simulation, 25(1), pp. 53-61.

Lee, J.Y., Nam, H.W., Jun, C.M., 2017. A study on evacuation model based on Floor Field Model considering fire spreading. Conference on Geo-Spatial Information 2017 Fall. pp.74-77.
McGrattan, K., Hostikka, S., McDermott, R., Floyd, J., Weinschenk, C., and Overholt, K., 2013. Fire Dynamics Simulator User's Guide. NIST, USA.

Nam, H.W., 2012. Developing a cellular automata-based pedestrian model incorporating microscopic behavior. Ph.D. Thesis, University of Seoul, Seoul, Korea. 2-3pp.

Nam, H.W., Kwak, S.Y., and Jun, C.M., 2016a. A study on building evacuation simulation using Floor Field Model. Journal of the Korea Society for Simulation, 25(2), pp. 1-11.

Nam, H.W., Kwak, S.Y., and Jun, C.M., 2016b. A study on comparison of improved floor field model and other evacuation models. Journal of the Korea Society for Simulation, 25(3), pp. 41-51.

NIST, 2017. Fire Dynamics Simulator (FDS) Software, Version 6.6.0. Open Source https://pages.nist.gov/fds-smv/down loads.html (2 Nov 2017).

Rie, D.H., 2008. A Study on the Development of Performance Based Fire Risk Assessment Program. Journal of the Korean Society of Safety, 23(5), pp. 1-6.

Wang, C., and Wang, J., 2016. A Modified Floor Field Model Combined with Risk Field for Pedestrian Simulation. Mathematical Problems in Engineering, 2016, pp. 1-10.

Zheng, Y., Jia, B., Li, X. G., and Jiang, R., 2017. Evacuation dynamics considering pedestrians' movement behavior change with fire and smoke spreading. Safety Science, 92, pp. 180-189.

Revised August 2018 